Kompass

Dermatologie

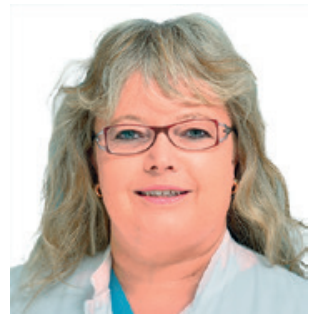

\section{Bettina Wedi}

Klinik für Dermatologie, Allergologie und Venerologie, Medizinische Hochschule Hannover (MHH), Hannover, Deutschland

\title{
Allergologie - wichtiger und spannender denn je!
}

In sehr kurzer Zeit hat sich durch die Corona-Pandemie nicht nur unser aller Alltag und die ärztliche Tätigkeit allgemein stark verändert. Sehr konkret gibt es auch neue Anforderungen an Allergologen und Dermatologen.

Seit Beginn der Verabreichung verschiedenster SARS-CoV-2-Impfstoffe vergeht kein Tag ohne multiple Kontaktanfragen hinsichtlich der allergologischen Risikoeinschätzung in Bezug auf eine zukünftige Impfung. Nur die wenigsten Vorerkrankungen bergen nach derzeitigem Kenntnisstand tatsächlich ein Risiko, passend zum Statement «Impfrisiko und das noch größere Risiko, nicht geimpft zu sein». In diesem Schwerpunktheft Allergologie finden Sie die aktuellen allergologischen Empfehlungen, hochkompetent kommentiert von Prof. Dr. Vera Mahler vom Paul-Ehrlich-Institut. Mit der Verfügbarkeit der SARS-CoV-2-Impfstoffe haben wir zunehmend mit Patientinnen und Patienten zu tun, die auf die erste oder zweite Impfung mit einer «allergischen» Symptomatik regiert haben. Allergische Reaktionen sind nicht immer einfach von nicht-allergischen Impfreaktionen wie beispielsweise vasovagalen Synkopen oder stressbedingtem Flush zu unterscheiden. Diese Problematik kennen wir schon aus jahrzehntelanger Erfahrung bei der Abklärung von Lokalanästhetika-Allergien mit le- diglich an einer Hand abzählbaren Einzelfällen einer bestätigten Allergie pro allergologischem Zentrum. Dass trotz dieser Erkenntnisse die Abklärung von Lokalanästhetika-Überempfindlichkeiten weiterhin zum allergologischen Alltag gehört, sollte nachdenklich machen. Eine diesbezügliche Leitlinie mit Einbindung der Deutschen Gesellschaft für Zahn-, Mund- und Kieferheilkunde (DGZMK) könnte zu einem Umdenken führen.

$\mathrm{Ob}$ Inhaltsstoffe wie Polyethylenglycol (PEG) 2000, Tromethamin oder Polysorbat 80 in SARS-CoV-2-Impfstoffen tatsächlich verantwortlich sind für allergische oder pseudoallergische Reaktionen, wird die nähere Zukunft zeigen. Es könnte aufgrund der teilweisen Verbreitung auch in anderen Pharmazeutika, Kosmetika und Lebensmitteln weitreichendere Konsequenzen haben. Die Comprehensive Allergy Center und andere Allergiezentren befassen sich aktuell mit dieser Thematik und stehen in einem engen Austausch. Zum jetzigen Zeitpunkt lässt sich sagen, dass die Häufigkeit anaphylaktischer Reaktionen auf SARS-CoV-2-Impfstoffe sich mit zunehmender Zahl verabreichter Impfdosen eher relativiert und sich aktuell auf einem Niveau von etwa 0,002\% eingependelt hat. Der sogenannte «COVIDArm», eine verzögerte lokale kutane Überempfindlichkeit, etwa eine Woche nach
Impfung mit einem mRNA-Impfstoff, ist wesentlich häufiger, aber kein Grund auf eine 2. Impfung zu verzichten.

Wir Dermatologen und Allergologen müssen darauf achten, neben den täglich auf uns einprasselnden neuen Informationen und Regeln hinsichtlich Corona die eigentlichen Schwerpunkte unserer Tätigkeit und diesbezügliche Neuigkeiten nicht aus den Augen zu verlieren. Die Zahl neuer klinischer Studien im Bereich der Dermato-Allergologie ist beachtlich. Die Ergebnisse haben teilweise bereits zu einer Revolutionierung des Verständnisses der Haut-Immunologie und der Allergologie geführt, aber auch zahlreiche neue Fragen aufgeworfen. Es ist interessant zu sehen, dass einzelne Substanzen in mehreren Indikationen geprüft werden, die nach derzeitigem Kenntnisstand eigentlich eine ganz unterschiedliche Pathophysiologie aufweisen. Es ist eine Besonderheit, dass einige Präparate zunächst im dermatologisch-allergologischen Bereich und erst nachfolgend hinsichtlich ihrer Wirksamkeit auf andere Organsysteme (Lunge, Nase) geprüft wurden. Zukünftig wird in jedem Fall die Frage der Finanzierung der hochpreisigen modernen Biologika- oder small molecule-Therapien zu klären sein. Seit Jahrzehnten bewährte Therapieprinzipien, insbesondere topische Therapien und Fototherapien, sollten unbedingt weiter beherrscht und 
verwendet werden. Nicht jeder Patient benötigt und wünscht eine vielleicht lebenslange Systemtherapie.

Innovative Therapieansätze, die nicht von der Industrie initiiert sind, benötigen Förderung. Wie wichtig unabhängige universitäre Forschung ist, hat auch die SARS-CoV2-Impfstoffentwicklung gezeigt. Die Deutsche Gesellschaft Allergologie und klinische Immunologie fördert daher Forschungsprojekte wie das von PD Dr. Julia Esser-von Bieren, das 2020 mit dem Forschungsförderpreis der DGAKI ausgezeichnet wurde in diesem Heft noch einmal vorgestellt wird.
Wie die digitale Transformation im allergologischen Alltag angekommen ist, kommentiert Dr. Stephan Traidl und Dr. Dorothea Wieczorek analysiert aktuelle Daten zur Insektengiftanaphylaxie aus dem Anaphylaxie-Register (ANAPHYLAXIE.net), das mittlerweile beachtliche über 11000 abgeschlossene Fälle, davon mehr als 8000 aus Deutschland, aufweist. Bei Allergologen ist außerdem detektivischer Spürsinn gefragt. Ein Update neu zu beachtender Kontaktallergene, wie in der Übersichtsarbeit von Uter et al. präsentiert, ist daher sehr willkommen für die alltägliche Arbeit.
Besonders spannend ist auch die sehr gut allergologisch aufgearbeitete Fallpräsentation einer Zitronenkernallergie. Wer von Ihnen hätte dies als Auslöser einer Anaphylaxie nach Salatgenuss vermutet?

Dies alles und noch viel mehr finden Sie in diesem Schwerpunktheft Allergologie.

Ich wünsche Ihnen viel Spaß bei der Lektüre - das Heft ist nicht ganz, aber überwiegend coronafrei!

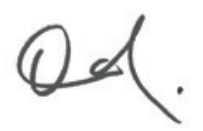

Ihre Prof. Dr. med. Bettina Wedi 